\title{
Rire pour ne pas pleurer
}

Étude du roman polyphonique Le Voleur de Bible de Göran Tunström

\section{Annelie Jarl Ireman}

\section{(2) OpenEdition}

\section{Journals}

Édition électronique

URL : http://journals.openedition.org/aes/395

DOI : 10.4000/aes.395

ISSN : 2258-093X

Éditeur

Laboratoire LISAA

Référence électronique

Annelie Jarl Ireman, «Rire pour ne pas pleurer », Arts et Savoirs [En ligne], 3 | 2013, mis en ligne le 15 février 2012, consulté le 19 avril 2019. URL : http://journals.openedition.org/aes/395 ; DOI : 10.4000/ aes.395

Ce document a été généré automatiquement le 19 avril 2019.

Centre de recherche LISAA (Littératures SAvoirs et Arts) 


\title{
Rire pour ne pas pleurer
}

\author{
Étude du roman polyphonique Le Voleur de Bible de Göran Tunström
}

\author{
Annelie Jarl Ireman
}

1 Le choix du roman Le Voleur de Bible pour illustrer l'adaptation comique peut paraitre étonnant. En effet, il est difficile de trouver une histoire plus tragique que celle-ci. Après une enfance difficile, vécue dans la pauvreté matérielle et spirituelle, le protagoniste, Johan, découvre le monde des livres et le grand projet de sa vie : voler La Bible d'argent. Le titre de la traduction française évoque cette Bible, unique document existant rédigé en gothique, qui se trouve à l'Université d'Uppsala. Pour la voler, il doit étudier, ce qui le mènera à écrire une thèse de doctorat. Cependant, il abandonne l'amour de sa vie, sa cousine Hedvig, qui en perd la raison. Avant de mourir, elle donne naissance à leur fils, à qui le livre s'adresse. En prison (où il se trouve pour avoir volé un manuscrit), Johan écrit à ce dernier pour lui expliquer ses actions et en être jugé. Ce roman est considéré comme l'œuvre la plus noire de Tunström, mais aussi, paradoxalement, comme la plus drôle. Certains la trouvent insupportable à lire tant elle est triste, d'autres en apprécient l'humour. Entre ces deux extrêmes se trouvent ceux qui lisent l'œuvre remplis de compassion mais que l'humour tient à distance d'un naufrage en compagnie des personnages. Cet aspect comique qui illumine l'œuvre apparaît en partie grâce au jeu intertextuel.

2 Göran Tunström fait sans cesse appel à la culture du lecteur. Nous découvrons avec plaisir certaines des allusions plus ou moins cachées. Or le sourire intellectuel n'est pas le plus important, l'auteur pousse le lecteur à aller plus loin, à découvrir davantage d'aspects dans ses textes. L'intertextualité donne à l'œuvre une nouvelle dimension, contribue à sa structure et à son sens. Le Voleur de Bible est un exemple de narration figurale, le lecteur devant notamment, à l'aide de l'intertextualité, interpréter le discours réaliste, le "déréaliser ", pour accéder à sa dimension allégorique. On peut aussi parler de «roman mythologique » ${ }^{1}$ quand un schéma déjà existant d'événements et de personnages tirés de la mythologie ou de la littérature se trouve dans une histoire nouvelle. Les personnages agissent ainsi en conformité avec leur prédécesseur mythique ou littéraire. Nous allons voir comment trois hypotextes, trois schémas, le premier littéraire, le deuxième biblique 
et le troisième mythique, sont présents dans ce roman. Il s'agit du conte de fée, du Livre d'Ézéchiel et du mythe d'Orphée et Eurydice².

3 Notons d'abord que les noms des personnages sont des allusions. Johan porte le surnom de Johan de la Cruche parce qu'il vend du vin aux étudiants pour gagner sa vie, mais c'est également une allusion au mystique espagnol du $\mathrm{XVI}^{\mathrm{e}}$ siècle Jean de la Croix, qui a essayé de transformer la pierre en or. En suédois la ressemblance entre les deux noms est frappante: Johannes av Korset pour Jean de la Croix, et Johan av Kruset pour Johan de la Cruche. Ce dernier, lui, a le don de transformer de l'eau en vin. «Et ce mystère de la transformation n'avait jamais cessé de m'étonner. Transformer le granit en or à force de l'arroser patiemment [...], franchir constamment des seuils ${ }^{3}$ ! Nuit obscure de Jean de la Croix incite l'homme à se libérer de son moi et à sortir dans la nuit pour trouver la personne aimée (Dieu) ${ }^{4}$. Or le Johan de Tunström, en sortant dans la nuit, se perd. Il s'isole concrètement dans sa maison et mentalement dans son moi, échouant ainsi à aller à la rencontre de sa personne aimée qui est Hedvig. Cette dernière porte le même prénom que la fille du Canard sauvage de Henrik Ibsen qui est repoussée par son père parce qu'elle n'est pas sa propre fille. ${ }^{5}$ Elle ne parvient pas à tuer son canard pour montrer à son père qu'elle l'aime plus que tout et, à la place, elle se suicide. La Hedvig de Tunström a également un oiseau, le "corbeau de miséricorde ${ }^{6}$ ». Comme le personnage d'Ibsen, son oiseau devient en quelque sorte une partie d'elle. Elle sait ce qu'il pense et il parle à travers elle. L'oiseau lui donne la sécurité que ses parents sont incapables de lui procurer. Mais elle tue son corbeau contrairement à la Hedwige du Canard sauvage. Peu après, elle sombre dans la folie, qui précède son véritable suicide. Hedvig symbolise également le canard de la pièce d'Ibsen. Elle n'a que quatre orteils sur chaque pied, comme les oiseaux n'ont que quatre doigts aux pattes, et elle est sacrifiée, tout comme le canard sauvage.

\section{Une polyphonie littéraire}

Chez Tunström, différentes voix portent ensemble la narration de l'histoire. L'un des personnages, le je principal du roman, raconte son histoire mais laisse souvent sa place à un narrateur omniscient et extérieur à l'histoire, ou bien à un autre personnage. Dans Le Voleur de Bible, la narration se fait à la troisième personne pendant 150 pages ${ }^{7}$, c'est-à-dire pendant la moitié du livre, jusqu'au moment où Johan admet enfin être le narrateur et où le récit passe de la troisième personne à la première. L'utilisation de la troisième personne donne un point de vue plus objectif et permet de regarder les événements de l'extérieur. Johan voudrait garder cette distance mais son histoire n'est pas objective. À partir du moment où il le comprend, il parle en son nom propre. Il explique :

Jour et nuit j'ai rédigé, moi que l'on nomme Johan de la Cruche, la saga de ma vie avec la constante impression que le temps presse. J'ai essayé de conserver du recul en me nommant à la troisième personne. Ce n'est plus possible. Car c'est moi, personnage ridicule, qui suis penché au-dessus d'Hedvig, persuadé que je peux lui faire réintégrer le monde des conventions ${ }^{8}$.

Nous trouvons des indices de l'identité véritable du narrateur avant ce passage. Déjà à la dix-neuvième page, ce narrateur qui semble extérieur à l'histoire se trahit une première fois en écrivant « je », mais on ne sait pas encore qui il est ${ }^{9}$. Et une centaine de pages plus loin, nous avons une première indication que c'est effectivement Johan, le personnage principal, qui est en train de raconter son histoire en cachant sa véritable identité au lecteur ${ }^{10}$. Le lecteur finit par comprendre que Johan construit le roman en racontant son 
histoire. Il s'agit donc d'un discours métanarratif. Johan passe ainsi du rôle de héros à celui de héros-narrateur puis au rôle de héros-narrateur-écrivain fictif. La création artistique prend la forme d'une confession, dans laquelle le narrateur s'adresse à un narrataire fictif, son fils, qu'il ne connaitt pas. Le fait qu'il l'appelle « Le Juge » montre bien qu'il veut être jugé par lui, et pardonné. Ce narrataire est aussi le lecteur, qui doit juger le comportement de Johan. Pour reconstruire le passé, il utilise des documents (fictifs mais ayant l'air réels), ce qui permet à l'écrivain de faire entendre des voix du passé. Nous trouvons des lettres écrites notamment par Hedvig et Ida, sa mère, lues par le narrateur. Il cite même une de ses propres lettres adressée à Hedvig, qu'il a en sa possession parce qu'elle la lui a été retournée. Il peut donc être sûr de ce qu'il a écrit il y a longtemps. Johan utilise aussi ses propres notes retrouvées, ce qui lui permet de se souvenir des événements et de ce que d'autres ont dit, puisqu'il l'a noté sur le moment. Ces documents permettent une narration à la deuxième personne, puisque la plupart s'adressent au narrateur. L'histoire de Johan est donc racontée de son propre point de vue à la première personne, de celui d'un narrateur omniscient à la troisième personne, et du point de vue des personnages à travers leurs lettres à la deuxième personne, ce qui accentue l'aspect réaliste du roman.

Dans une partie du livre, un autre personnage devient le narrateur de sa propre histoire. Il s'agit de Wiljarith, l'auteur d'un manuscrit que Johan a trouvé et «emprunté » à Ravenne ${ }^{11}$ et qu'il utilise pour ses recherches ${ }^{12}$. C'est une copie (fictive bien sûr) de La Bible d'argent, commandée par le roi des Goths Théodoric. Ce dernier ne sait pas lire, mais désire quand même une œuvre magnifique qui restera après sa mort. De la même manière, les recherches et la Bible d'argent ne représentent, aux yeux de Johan qu'un trésor capable de changer sa vie. Mais en perdant ce qui fait le prix de l'existence, l'amour, sa vie ne devient qu'un ornement vide de sens. Ce passage joue donc le rôle de miroir dans lequel se reflète l'histoire principale. La situation de Johan ressemble en effet beaucoup à celle de Wiljarith quatorze siècles plus tôt. C'est un savant infirme comme Johan. Son épouse, comme Hedvig, ne parle presque pas. Wiljarith consacre sa vie à un projet sans aucun sens, car une fois terminée, l'œuvre ne pourra être lue par personne, puisque sa langue est morte. Il sacrifie même sa femme à ce projet et utilise, après son décès, sa peau comme parchemin. De la même façon, Johan sacrifie Hedvig pour accomplir son projet. En écrivant ses confessions à son juge, il est conscient que son projet était vain, qu'il l'a empêché de vivre, qu'il lui a volé sa vie. Le titre de la version originale est Tjuven, ce qui signifie «le voleur». Ce voleur n'est pas tant Johan que ce projet $^{13}$. Le manuscrit de Ravenne est donc intégré dans le roman pour donner des renseignements sur La Bible d'argent et son auteur, mais surtout pour jouer comme texte reflet et ainsi jeter une nouvelle lumière sur la vie de Johan.

7 Tunström laisse également la parole à d'autres personnages se servant d'une narration polyphonique. Ce terme musical signifie qu'il y a plusieurs voix qui sont combinées suivant certaines règles, comme c'est le cas dans la musique de Bach à laquelle l'auteur fait souvent référence, notamment dans le roman L'Oratorio de Noë $l^{14}$. Si Tunström utilise lui-même le terme de polyphonie, il fait, par là, avant tout référence au jeu relationnel du philosophe allemand Martin Buber $(1875-1965)^{15}$ car il veut écrire un roman polyphonique pour pouvoir analyser les relations. La polyphonie littéraire signifie selon Bakhtine qu'un roman contient plusieurs voix, à savoir des personnages avec des consciences indépendantes, que l'écrivain écoute et laisse agir en prenant une position anti-autoritaire dans le but d'entrer en relation avec eux. Ce dialogue se passe dans un 
maintenant, car l'auteur doit se trouver dans la même dimension de temps que ses personnages ${ }^{16}$. Le lecteur doit être très attentif aux changements du discours narratif et, de plus, il doit juger le contenu de vérité (fictionnelle) de ce qui est dit, puisque les différentes voix donnent parfois différentes versions. ${ }^{17}$

Dans cette narration alternée, Tunström se sert également d'autres voix que celles des personnages proprement dits, venant d'autres époques. La polyphonie est ainsi renforcée par l'intertextualité. La chronologie et la distance disparaissent et toutes ces voix s'harmonisent dans l'œuvre et agissent simultanément tout en gardant chacune une certaine indépendance. Dans Le Voleur de Bible, l'auteur joue notamment avec trois hypotextes, qui nous aident à comprendre la relation entre les deux personnages principaux.

\section{Le conte de fées}

9 L'histoire d'amour entre Johan et Hedvig est ponctuée par des références aux contes de fées, à leurs princes et leurs princesses. Traditionnellement, les contes de fées se terminent bien, ce qui n'est pas le cas du roman de Tunström. Ici, le conte donne un contrepoids heureux qui renforce l'idée d'échec mais apporte en même temps un aspect comique. Johan veut être un prince qui sauve sa princesse. À plusieurs reprises, il parle d'un château. «Longtemps j'avais cru que, maintenant que j'avais atteint l'orée, nous allions nous prendre par la main et courir ensemble vers des prés, des châteaux et la liberté $^{18}$.» Le but de ses études est de réussir dans la vie, pour pouvoir offrir une meilleure existence à Hedvig, c'est-à-dire lui donner le château qu'elle a mérité après une enfance misérable. Hedvig est Cendrillon, qui a dû s'occuper de la maison, de ses frères et de ses sœurs. Certes, la vraie Cendrillon n'a pas de mère et Hedvig en a une, Ida, mais celle-ci est souvent absente. Même présente, Ida n'a pas la capacité de protéger les enfants de leur père violent, ce que Hedvig doit faire à sa place. En essayant de protéger sa mère contre les viols répétés du père, Hedvig s'y expose d'autant plus. Toute cette souffrance et le poids de ces responsabilités trop grandes pour une jeune fille la mènent à l'hôpital psychiatrique. Enfermée dans l'asile, comme une princesse dans une tour de château fort, elle attend son prince. Mais elle doit l'attendre très longtemps. Malgré les lettres qu'elle lui envoie, Johan ne vient pas, ce qui l'oblige à changer le cours du conte, à s'enfuir de sa prison et à partir à la recherche de son prince.

10 Johan ressemble, selon Hedvig, à une grenouille. Il est effectivement infirme, petit et faible, au visage extrêmement laid, et il n'a pas le courage du prince. Elle est donc consciente qu'il n'est pas un prince, mais, étant une grenouille, il est aussi capable de se transformer. Grâce à un baiser, la transformation pourrait avoir lieu. Or Johan n'est pas un prince, pas même un prince déguisé, mais un homme, avec des défauts. Il ne peut pas remplir les exigences de sa princesse, ce dont finalement, Hedvig se rend compte :

Hedvig riait.

- Sauver la grosse Hedvig du loup, non mais! Lui offrir un château et de beaux

habits ! Vivre avec une grenouille dans le château ${ }^{19}$ !

11 Elle sait maintenant que Johan ne sera jamais qu'une grenouille. Il faut dire que Hedvig n'a rien d'une princesse traditionnelle : elle est obèse, elle bave, se gratte jusqu'au sang et son regard est empli de mépris et de répugnance. Dans cette citation, nous observons la présence du loup, symbole du danger et du mal dans les contes, et, une fois encore, le château, symbole de la vie heureuse. Hedvig est toujours prisonnière du loup et le château 
est définitivement hors de portée. Hedvig et Johan se rejoignent à la fin du livre dans un acte sexuel mais sans que cette rencontre soit entière, parce qu'ils n'échangent même pas le baiser nécessaire à la transformation. Johan reste une grenouille et Hedvig reste seule et prisonnière. L'aspect comique de cette scène tragique vient entre autres du fait que les rôles sont inversés : ce n'est pas le prince qui vient sauver la princesse, c'est Hedvig qui essaie de réveiller un Johan ivre mort par des chants de solitude. Ce n'est pas le soi-disant prince Johan qui l'embrasse tendrement, c'est elle qui le réveille en le séduisant. Nous sommes finalement très loin du conte de fées.

12 Johan et Hedvig suivent dans le roman un mouvement vertical dans lequel le point abyssal est représenté soit par les Enfers de la mythologie soit par la vallée des Ossements du Livre d'Ézéchiel dans l'Ancien Testament. Dans le chapitre 37 de ce livre, le prophète est placé par Dieu dans une plaine remplie d'ossements, qui le confronte à la mort avant de rendre possible le retour à la vie : «La main de l'Éternel fut sur moi, et l'Éternel me fit sortir en esprit et me déposa dans le milieu de la vallée; celle-ci était remplie d'ossements. Il me fit passer auprès d'eux, tout autour ; or, ils étaient très nombreux, à la surface de la vallée, et très secs. Il me dit: Fils d'homme, ces os pourront-ils revivre? Je répondis: Seigneur Éternel, c'est toi qui le sais ${ }^{20}$ ! Le prophète réussit à créer une cohérence dans le chaos: les os se rassemblent, les corps reprennent vie. La campagne pleine d'ossements symbolise, chez Tunström, la réalité qui n'est pas encore structurée ${ }^{21}$. Cette vallée est chez Tunström évoquée par des références à la plaine, au vent et à la boue ${ }^{22}$. Le narrateur a le même rôle qu'Ézéchiel et doit donc se trouver, comme lui, au fond de l'existence, afin de pouvoir remonter et reconstruire. Le lien avec Ézéchiel illustre donc le mouvement qui va du chaos jusqu'à la vie et la vallée des Ossements représente le point ultime inférieur atteint par les personnages. Ézéchiel se trouve en exil, loin de Jérusalem. De même, Johan se trouve loin de Sunne, le bourg de son enfance et le point central de son existence. Il cherche à rétablir l'ordre par la force de la narration, c'est-à-dire à rassembler les ossements desséchés. Hedvig se trouve dans la vallée des morts, dans sa maladie, où, au début, elle attend Johan, qui doit la ressusciter. Tandis que Hedvig y séjourne depuis longtemps, Johan, lui, y tombe régulièrement mais il réussit à remonter ensuite. Sa vallée des Ossements est le Trou de Boue, l'endroit où Johan se détruit le dos en sauvant son père. C'est un grand trou rempli de déchets et de boue à côté de la maison de son enfance. À l'âge adulte, il retombera dans ce trou chaque fois qu'il perdra pied.

Celui qui sait tout abandonner saura tout vivre. Et je le fis, pour la énième fois, pris la direction du précipice profond du Trou de Boue. Je voulais rouler dans les miasmes et les ferrailles du tas d'ordures. Mais le temps me l'avait subtilisé : autour de moi je ne voyais que la plaine et le vent de la plaine et son épouvantable Maintenant ${ }^{23}$.

13 À la fin du livre, Johan retrouve enfin Hedvig: «J'aperçus l'être étrange loin sur l'océan de terre et restai à la lisière du bois, ne voulant pas abîmer mes chaussures plus que nécessaire, je voulais simplement voir. Le vent soufflait fort $[. . .]^{24} »$ La jeune fille se trouve alors dans ce champ boueux battu par le vent: toujours la plaine d'Ézéchiel. Or Johan n'est pas capable de lui redonner vie car il n'a pas le pouvoir du prophète.

\section{Orphée et Eurydice}

Le mythe d'Orphée et Eurydice est sans doute l'hypotexte le plus fréquent dans l'œuvre de Tunström. Orphée, qui refuse d'accepter la mort d'Eurydice, la rejoint afin de 
persuader les puissances des Enfers de la laisser repartir. Grâce à sa musique, il peut obtenir ce qu'il désire mais doit promettre de ne pas regarder sa femme avant d'être revenu à la lumière du jour. Cependant, juste avant d'arriver, Orphée ne peut plus résister, il se retourne, la regarde et la perd pour toujours ${ }^{25}$. Le Voleur de Bible montre explicitement ses liens avec le mythe, dans la mesure où chacun des trois livres du roman débute par un extrait du poème « Orphée. Eurydice. Hermès » de Rainer Maria Rilke ${ }^{26}$.

Le livre premier commence par la citation du début du poème de Rilke. Dans cette partie du poème, Orphée avance pour sortir des Enfers suivi d'Eurydice et d'Hermès. Orphée veut se retourner parce qu'il ne sait pas si elle le suit vraiment, mais il n'ose pas. Dans cette première partie du roman, Hedvig sombre dans la folie et Johan ne peut plus l'atteindre. Il la supplie de revenir :

- Reviens, reviens-moi. Tu es la seule de tous qui compte pour moi. Il ne faut pas que tu disparaisses.

- Si, je crois qu'il faut que je le fasse. [...] C'est certainement ce qui était décidé27.

Elle doit suivre le schéma du mythe mais elle est convaincue qu'il la fera revenir puisqu'il est Orphée. Ensuite elle est enfermée à l'hôpital et Johan, par désespoir, fait une première descente aux Enfers, le Trou de boue dans lequel est tombé son père ivre mort. Il appelle sa bien-aimée dans le noir, mais ne la trouve pas. Johan rend visite à Hedvig à l'asile ; là, bien que partie "pour le bout du monde ${ }^{28}$ ", dans un moment de lucidité elle le supplie de la faire sortir de là. Johan répond : « Un jour je te sortirai d'ici. Je dois seulement ${ }^{29}$... »

Le livre deuxième commence dans la prison où Johan écrit, reconstruit son monde à lui. Dans cette partie, il quitte Sunne pour étudier à Uppsala. Il conçoit ses études comme « un moyen pour charmer Hedvig et l'attirer hors des ténèbres ${ }^{30}$ ", comme il va le dire plus tard. Cette partie du poème de Rilke parle d'Eurydice qui avance toujours mais qui ne pense plus à Orphée. Elle se trouve dans le monde des morts, ne comprend rien de ce qui se passe. Hedvig va également commencer son voyage vers la vie mais elle est tout aussi perdue dans un monde à part. "Au même moment Hedvig passe le portail de l'hôpital. Elle marche toute la nuit ${ }^{31}$.» Comme Orphée, Johan est censé sauver sa bien-aimée des Enfers mais il l'oublie pendant ses études et ce qui devrait la sauver la détruit. Lorsqu'il a enfin la permission de descendre dans la cave où est conservé le trésor national, il prend conscience de sa faute. En remontant dans l'ascenseur pour assister à la cérémonie qui suit sa soutenance, il sait qu'une partie de lui est restée aux Enfers, et qu'il n'a pas réussi à en sortir Hedvig :

Mais le Voleur, lui, restait en bas. Et je m'entendis moi-même répéter à Hedvig des années durant:

- Il faut simplement que je... Je vais d'abord ${ }^{32} \ldots$

Le livre troisième commence par «Elle n'était plus cette jeune femme blonde ${ }^{33} . .$. » Rilke nous dit à la fin du poème qu'Eurydice n'est plus la femme qu'elle était autrefois. Elle n'appartient plus à Orphée. Quand il la regarde, elle retourne sans regrets aux Enfers. Dans le monde tunströmien, plusieurs années ont passé et Johan se consacre à sa carrière universitaire, toujours dans le but vague de sauver Hedvig. Un jour il la découvre près de sa maison mais il la reconnaît à peine. Elle n'est plus la même femme : «[...] une personne grande et difforme marchant courbée sur la terre », «[...] on aurait dit une motte de terre ${ }^{34}$.» Dans sa folie, Hedvig a toujours l'espoir d'être sauvée. Elle le supplie de chanter la chanson. Son salut est encore possible grâce à la musique d'Orphée, mais Johan ne sait pas de quelle chanson elle parle. 
- Si tu promets de chanter la chanson, je viendrai.

- Quelle chanson? [...]

- Je ne veux suivre que celui qui connaît la chanson.

- Mais quelle chanson ${ }^{35}$ ? "Qui avais-je été pour me croire capable de la sortir des ténèbres en chantant?», se demande-t-il à la fin du livre ${ }^{39}$. Orphée réussit à entrer aux Enfers et c'est seulement après, qu'il n'arrive pas à accomplir sa mission. Johan trahit plusieurs fois. Il n'a d'abord pas la capacité de descendre là où il aurait pu la rencontrer. «Je ne pouvais même pas tomber ${ }^{40}$.» En réalisant que Hedvig ne veut plus être sauvée, qu'elle a rompu les liens avec lui, il comprend combien il a échoué dans son intention de trouver un sens à sa vie à travers son projet. Il ne lui reste plus, à lui, qu'à mourir. Johan se soûle alors dans l'obscurité de la cave, représentation allégorique de sa descente aux Enfers. Il est enfin prêt à la rencontrer. Mais les rôles sont dès lors inversés : c'est Hedvig qui le porte hors du sous-sol en chantant. La rencontre est à ce moment possible : «Et la condition est : ne pas être vue. Maîtriser la scène sans être surveillée. Sans être exigée par les yeux des autres, par leurs espoirs, leurs souvenirs ${ }^{41} \ldots$.. Mais Johan trahit une nouvelle fois: il regarde Hedvig d'un regard qui la juge :

Alors je la regardai. Au moment même où mon jet gicla en elle, j'ouvris les yeux et me fis présent. [...] Elle me vit voir. Elle me vit nous voir enfermés dans un grand cri. Voir ça, c'était l'obliger à retourner aux Enfers ${ }^{42}$.

Il transgresse la condition en la regardant, accomplissant ainsi le mythe. Hedvig doit rester aux Enfers, c'est pourquoi elle se suicide après la naissance de leur fils. Johan, lui, commence à remonter seul. La naissance du fils donne au roman un aspect positif. La recréation commence quand le tu de la narration est conçu. Johan comprend qu'il est coupable et fait ses aveux à son fils, c'est la seule possibilité de renaissance pour lui.

Johan est donc Orphée mais il ne ressemble pas beaucoup à son prestigieux modèle. Ce n'est pas un héros de la mythologie, il n'est ni beau, ni courageux. Selon Hedvig, c'est même " l'être le plus laid qui existe ${ }^{43}$. » Il n'entre pas aux Enfers grâce à son talent, il y tombe en se soûlant. Sa musique est une chanson grossière. Hedvig est une Eurydice folle et répugnante que Johan ne supporte pas de regarder.

Pour conclure, nous pouvons constater que l'œuvre de Tunström est ouverte vers d'autres œuvres littéraires, mythiques et religieuses. Grâce à l'intertextualité l'auteur se met en relation avec le lecteur bien sûr, mais aussi avec d'autres écrivains, ce qui contribue à rendre le roman polyphonique. L'œuvre devient universelle; elle invite le lecteur à voir ce qui est universel dans son destin personnel. L'auteur donne des clés d'interprétation au lecteur pour l'aider à comprendre le texte. Les personnages suivent 
un certain schéma mythique durant un moment, pour ensuite l'abandonner ou l'échanger contre un autre. Ainsi, Johan alterne les rôles de Jean de la Croix, d'Orphée, du prince grenouille, d'Ézéchiel, et encore d'autres. Le livre s'insère dans un univers culturel mais Tunström ne répète pas les histoires, il les réactualise à sa manière, les alterne. La transposition de ces histoires fondamentales de l'humanité dans un contexte nouveau et plutôt banal, voire vulgaire donne un aspect comique à l'histoire tragique qu'est Le Voleur de Bible. Dans le passage du registre noble au registre bas, la vallée d'ossements devient un vulgaire trou de boue, la princesse une jeune femme obèse à la recherche désespérée de son prince, le beau héros mythologique un infirme sans dons pouvant l'aider à accomplir ses projets. L'incapacité des personnages à agir comme leurs prédécesseurs et à remplir les conditions des hypotextes les rend ridicules. Par rapport aux personnages des contes de fées, des mythes et des livres bibliques, ceux de Tunström sont des caricatures, ils sont grotesques. Mais par rapport à la vraie vie, ne sont-ils pas plus authentiques? Il est malgré tout possible de s'identifier à ces personnages car dans leur fragilité, leurs faiblesses, ils sont humains. Le décalage donne une vision neuve de ces motifs, qui permet au lecteur d'aujourd'hui de mieux les comprendre. Pourquoi une princesse moderne ne pourrait-elle pas partir à la recherche de son bien-aimé au lieu d'attendre patiemment dans sa tour? Pourquoi un héros d'aujourd'hui n'aurait-il pas le droit d'être égoïste et de réaliser ses propres rêves avant de penser aux autres? Il ne s'agit pas d'une irrévérence de la part de l'auteur envers ses sources, mais d'une adaptation à un monde nouveau, un monde compliqué. L'auteur ne se moque pas du ridicule de ses personnages, il a de la compassion pour eux. Le comique, tout comme le tragique, fait partie de la vie. Johan, lui, a la capacité de rire de ses malheurs et nous rions avec lui, ce qui nous empêche de pleurer. Lire Le Voleur de Bible c'est s'égarer pendant un moment aux Enfers, aidé de l'humour qui, malgré tout, rend le voyage supportable, et même agréable.

\section{NOTES}

1. Terme emprunté à John. J. White, in Mythology in the Modern Novel: A study of Prefigurative Techniques, Princetown, New Jersey, 1971.

2. Pour une analyse plus approfondie de l'intertextualité dans l'œuvre romanesque de cet auteur, voir notre thèse Quête et intertextualité: Une étude thématique de l'œuvre en prose de Göran Tunström, Lille, Atelier National de reproduction des thèses, 2006, p. 241-345.

3. Göran Tunström, Le Voleur de Bible, traduit du suédois par Marc de Gouvenain et Lena Grumbach, in Euvres romanesques 1, Arles, Actes Sud, 1999, p. 655-656. Par la suite VB.

4. Jean de la Croix, Noche oscure, Paris, Gallimard/UNESCO, 1997.

5. Henrik Ibsen, Le Canard sauvage, Paris, Stock, 1944.

6. VB, op. cit., p. 538.

7. Jusqu'à la p. 617 dans CEuvres romanesques.

8. VB, op. cit., p. 617.

9. Ibid., p. 489.

10. Ibid., p. 532.

11. C'est la raison pour laquelle il se trouve en prison. 
12. VB, op. cit., p. 739-765.

13. Le titre de la traduction française nous mène donc dans une impasse.

14. Göran Tunström, L'Oratorio de Noël, traduit du suédois par Marc de Gouvenain et Lena Grumbach, Arles, Actes Sud, 1987.

15. Voir Martin Buber, Je et Tu, Paris, Aubier, 1969.

16. Michail M. Bakhtine, La Poétique de Dostoïevski, Paris, Éditions du Seuil, 1970, p. 82-117.

17. Pour une étude du discours narratif de l'œuvre de Tunström, voir notre thèse, op.cit., p. 30-81.

18. VB, op. cit., p. 779.

19. Ibid., p. 782.

20. La Sainte Bible, «Ézéchiel », 37 : 1-3, Paris, Alliance Biblique Universelle, p. 901.

21. Anders Tyrberg, Anrop och ansvar, Stockholm, Carlssons Bokförlag, 2002, p. 145-149.

22. Le mot suédois lera traduit par "boue» peut évoquer la terre sèche d'une plaine. Cet hypotexte joue un rôle encore plus important dans un autre roman de Tunström : La Parole du désert, traduit du suédois par Pascale Balcon, Arles, Actes Sud, 1991.

23. VB, op. cit., p. 690.

24. Ibid., p. 767.

25. Pierre Grimal, Dictionnaire de la mythologie grecque et romaine, Paris, Presses Universitaires de France, 1951, p. 332-333.

26. Rainer Maria Rilke, « Orphée. Eurydice. Hermès », Neue Gedichte, Leipzig, Insel-Verlag, 1907.

27. VB, p. 625 .

28. Ibid., p. 630.

29. Ibid., p. 631.

30. Ibid., p. 789. En suédois, c'est le mot pour Enfers (underjorden) qui est utilisé à la place des ténèbres.

31. Ibid., p. 691.

32. Ibid., p. 793.

33. Ibid., p. 717.

34. Ibid., p. 767.

35. Ibid., p. 768.

36. Ibid., p. 773.

37. Ibid., p. 512.

38. Ibid., p. 778.

39. Ibid., p. 783.

40. Ibid., p. 690.

41. Ibid., p. 784.

42. Ibid., p. 785.

43. Ibid., p. 782.

\section{INDEX}

Mots-clés : roman, polyphonie, rire, Tunström (Göran) 
AUTEUR

ANNELIE JARL IREMAN 